\section{Inversion of dorsoventral axis?}

SIR - It is widely believed that the ventral nerve cord of insects and the dorsal nerve cord of vertebrates evolved independently and at opposite positions in the animal ${ }^{1}$. Instead, we propose that the longitudinal nerve cords of insects and vertebrates derive from one and the same centralized nervous system in their common ancestor. Because the nerve cord is located ventrally in insects (classifying as Gastroneuralia ${ }^{2}$ ) and dorsally in vertebrates (Notoneuralia ${ }^{2}$ ), the ventral side of insects would then correspond to the dorsal side of vertebrates. This idea has often been discussed (beginning with Geoffroy StHilaire in the nineteenth century): it implies that the relative positions of the gut and nervous system, the location of the heart and the direction of the blood flow, are the same in insects and vertebrates ${ }^{1}$.

In accordance with this notion, insects and vertebrates appear to use similar genes during formation of their differently located nervous systems. For example, in Drosophila ${ }^{3}$ as well as in vertebrates ${ }^{4}$ the achaete-scute complex genes are expressed in neuronal precursor cells; that is, ventrally in insects and dorsally in vertebrates, where they seem to be involved in early neurogenesis ( $c$ in the figure).

In addition, non-tissue-specific but homologous genes are also expressed at opposite sides in Drosophila and in vertebrates (outside the nerve cord anlage): The decapentaplegic-related genes are involved in dorsoventral patterning in both insects and vertebrates. However, the Drosophila decapentaplegic gene is expressed dorsally and controls the differentiation of dorsal structures, whereas its vertebrate homologue, $B M P-4$, is expressed ventrally ${ }^{5}$ and has ventralizing activity $^{6}$ ( $c$ in the figure). Expression occurs within the ectoderm and the mesoderm, meaning that the dorsal or ventral expression is not tissue-specific or linked to any secondary cell rearrangements.

If the ventral side of insects corresponds to the dorsal side of vertebrates, how did this situation evolve? A rotation about the left-right axis (so that the dorsoventral and anteroposterior body axes are inverted, as implied in the protostome/ deuterostome concept ${ }^{2}$ ) can be excluded because the colinear expression patterns of Hox cluster genes indicate that the anteroposterior body axis was fixed before the evolutionary divergence of (protostome) gastroneuralians and (deuterostome) notoneuralians ${ }^{7}$. We therefore favour an inversion only of the dorsoventral axis.

We suggest that this inversion occurred during early chordate evolution, because
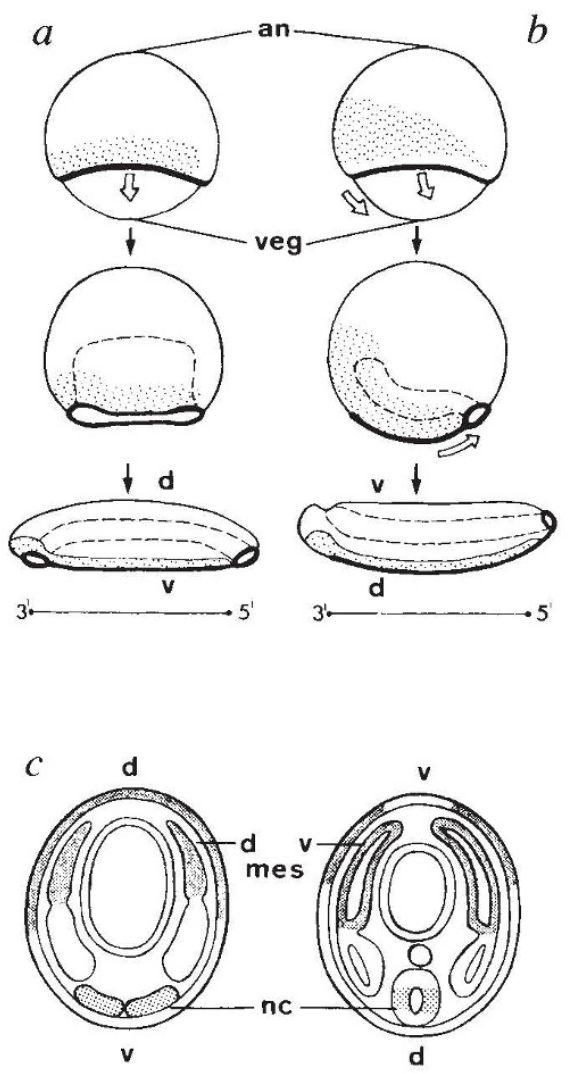

Location of future nerve cord (stippled) before gastrulation (upper panels), in late gastrula (middle) and after gastrulation (lower panels) in a schematic gastroneuralian animal ( $a$, annelid) and a notoneuralian animal ( $b$, Xenopus). Note that after gastrulation the sides bearing the nerve cord (dorsal in chordates and ventral in annelids) both cover the now internalized vegetal hemisphere (veg) of the developing embryo. Open arrows indicate movements of blastopore margin. Anteroposterior polarity is shown as $3^{\prime}-5^{\prime}$ with reference to Hox cluster gene expression. Bold lines, location of the (former) blastopore; broken lines, gut anlage; an, animal; d, dorsal; v, ventral. $a$, Gastroneuralian embryo showing an elongated blastopore and the ventral neurogenic region along its margins. Mouth and anus form from the anterior and posterior blastopore regions. In the developing trochophora larva of polychaete annelids the nerve cord develops along both sides of the elongated blastopore and comes to lie along the ventral side, which covers the former vegetal pole. $b$, Notoneuralian embryo (Xenopus). During gastrulation the anterior lip of the blastopore (left) moves posteriorly around the former vegetal hemisphere while, owing to mediad convergence of lateral cells, neural plate and notochord elongate towards the posterior until only the anus is left open at the neural plate stage (for example, in urodeles). Note that in deuterostomes the mouth forms at the new ventral body side, opposite the old gastroneuralian mouth; this might have occurred as a result of adult postures similar to that of living amphioxus. c, Gene expression patterns in schematic cross-sections in an insect (left) and a vertebrate (right), at about the phylotypic stage $^{7}$. Stippled region, achaete-scute complex genes $^{3.4}$ in the nervous system; shaded region, non-tissue-specific decapentaplegic-homologues ${ }^{6}$; nc, newe cord; mes, mesoderm. In Drosophila, decapentaplegic is expressed in the prospective dorsal and lateral ectoderm and in the dorsal mesoderm (mes) surrounding the embryonic midgut ${ }^{5}$; for better comparison the yolk is omitted and the as-yet gaping dorsal epidermis is shown closed dorsally. The vertebrate homologue of decapentaplegic, BMP-4, is expressed in the ventral mesoderm and in the ventral ectoderm (overlying the early limb buds) ${ }^{5}$. the deuterostome gastrulation in chordates can be conceptually derived from the gastrulation mode seen in the presumably older (protostome) gastroneuralians, such as the polychaete annelids. In polychaetes, which we assume to have more or less conserved the ancestral bilaterian mode of gastrulation, an elongated blastopore demarcates the future anteroposterior axis. Mouth and anus form at the anterior and posterior ends of this blastopore, while the nerve cord differentiates along its lateral margins ${ }^{2}$. The nerve cord and the future ventral side (the mouth-bearing side oriented towards the substrate) thus come to lie opposite the animal pole, where the vegetal hemisphere was located before gastrulation ( $a$ in the figure).

By contrast, in deuterostome chordates only the anus derives from the blastopore. Here the initially large blastopore is being reduced to a small opening at the animal's posterior pole, because the anterior blastopore lip progresses further around the vegetal hemisphere than do the lateral and posterior lips. While the anterior blastopore lip moves posteriorly, the future dorsal structures (neural plate and dorsal mesoderm) are being expanded along the anteroposterior axis and thereby gradually overgrow, from anterior to posterior, the former vegetal side of the chordate embryo ( $b$ in the figure). Thus if we consider a slit-like blastopore to be ancestral also for chordates, then the dorsal midline of the early neurula - towards which ectodermal and mesodermal cells converge during gastrulation ${ }^{8}-$ would mark the line along which the lateral blastopore lips had fused in the chordate ancestors. This finding indicates that the chordates turned upside down early in their evolution, and henceforth were carrying the nerve cord on their dorsal side, as first proposed in 1875 (ref. 9).

The proposed homology of nerve cords and the assumed inversion of the dorsoventral body axis in early chordates need not change the current view about genealogical relationships between the animal groups now considered 'gastroneuralians' and 'notoneuralians'. But it may change our view of what the common ancestor of insects and vertebrates could have looked like.

\section{Arendt}

\section{K. Nübler-Jung}

Institut für Biologie I (Zoologie), Albert-Ludwigs-Universität Freiburg, 79104 Freiburg, Germany

\footnotetext{
1. Romer, A. S. \& Parsons, T. S. The Vertebrate Body (Saunders, Philadelphia, 1977).

2. Fioroni, P. Rev suisse Zool 87, 261-272 (1980)

3. Martin-Bermudo, M. D. et al. Development 113, 445-454 (1991).

4. Zimmerman, K. etal. Development 119, 221 (1993).

5. Jones, C. M. et al. Development 111, 531-542 (1991)

6. Jones, C. M. et al. Development 115, 639-647 (1992).

7. Slack, J. M. W. et al. Nature 361, 490-492 (1993).

8. Keller, R. etal. Devi Dyn. 193, 199217 (1992).

9. Nübler-Jung, K. \& Arendt, D. Roux Arch. dev. Biol. 203

356 (1994).
} 